\title{
Texte et légendes de « Chim »
}

\section{L'ITALIE MÈNE LA BATAILLE CONTRE L'ANALPHABÉTISME - 1950 -}

2 - Vue d'ensemble de Rogiano Gravina. Le village de Rogiano Gravina est situé à environ quarante kilomètres au nord de Cosenza, au centre d'une province agricole très pauvre. Avec près de 6000 habitants, c'est la communauté la plus active de Calabre dans le domaine de la lutte contre l'analphabétisme. Un millier d'enfants d'âge scolaire vont à l'école, avec pratiquement $100 \%$ de présence, et 700 adultes fréquentent tous les soirs une école créée par Unione Per La Lotta Contro l'Analfabetismo avec le soutien du gouvernement. Ces quatre dernières années, le pourcentage d'analphabètes est tombé de 68 à $22 \%$. Cette action est supervisée par le Centre de culture du peuple dirigé par Giuseppe Zanfini, qui assure en même temps la fonction d'inspecteur pour la région de Calabre de l'Unione. Le Centre est devenu le cœur de la vie du village. Son mode de gestion est intéressant : il donne aux plus jeunes la possibilité de travailler dans un atelier rudimentaire où ils assemblent le matériel pédagogique, et où on enseigne la sculpture sur bois et des connaissances de base en menuiserie. Les femmes y apprennent les travaux manuels, la broderie et leurs heures d'école du soir sont réparties entre la couture et les leçons.

Rogiano Gravina, comme toute autre ville ou village en Italie, est amèrement divisée par des luttes politiques. Mais le Centre de culture du peuple a réussi à maintenir son statut apolitique. Communistes et démocrates-chrétiens sont assis côte à côte sur les bancs et font de gros efforts pour apprendre. La misère est celle de tous, et quand la vague d'occupation des terres a récemment submergé l'Italie du sud, Rogiano Gravina a offert l'extraordinaire spectacle d'un défilé réunissant les communistes et leur drapeau rouge, et les démocrates-chrétiens et leur drapeau blanc qui s'en allaient occuper ensemble la terre en friche du Baron Campagna, un propriétaire local possédant plus de 5000 hectares. Après de dures années de lutte contre l'analphabétisme, Rogiano Gravina nourrit de nouveaux espoirs. Le gouvernement construit une grande école élémentaire qui va remplacer les 42 salles de classe privées, dispersées dans le village. Un lopin de terre appartenant au village a été récemment attribué au Centre, qui a le projet d'y construire un nouveau bâtiment comprenant des salles de classe et des ateliers pour adultes. Mais ils ont besoin de fonds, et ils espèrent que l'UNESCO va les aider. Le coût du bâtiment a été estimé à 12000000 de lires, soit un peu moins de $20000 \$$. Cette somme prend naturellement en compte le travail bénévole de tout le village.

La photo a été prise du haut de la colline où sera construit le nouveau bâtiment scolaire. 
1. LUTTE CONTRE L'ANALPHABÉTISME. Au Centre d'éducation pour adultes de Rogiano Gravina, dans la province de Calabre, en Italie du sud, un paysan âgé termine sa première leçon d'écriture. 700 adultes fréquentent les écoles du village tous les soirs après toute une journée de travail.

4 - Un lopin de terre sur une colline surplombant Rogiano Gravina, récemment attribué au Centre par la mairie.

6 - Paysan âgé pendant sa première leçon d'écriture. Après un travail réussi auprès des jeunes paysans, le Centre s'efforce d'attirer les femmes et les personnes âgées.

2. LUTTE CONTRE L'ANALPHABÉTISME. La main d'un paysan âgé. Maniant maladroitement la plume, il essaie pour la première fois de sa vie de tracer les lettres de l'alphabet.

5 - Une classe pour personnes âgées au Centre de culture du peuple de Rogiano Gravina.

15 - Leçon d'écriture pour un paysan âgé au Centre de Rogiano Gravina, avec à l'arrière-plan une carte de la Calabre.

21 - Gros plan sur les mains d'un paysan âgé. Centre de Rogiano Gravina.

27-28 - Cours du soir au Centre de Rogiano Gravina. Certains paysans doivent faire huit kilomètres à pied depuis chez eux pour assister au cours.

33 - Leçon de géographie au cours du soir du Centre de Rogiano Gravina.

3. LUTTE CONTRE L’ANALPHABÉTISME. Avec Emmanuel Spingheri, son assistant, Giuseppe Zanfini (à droite), député-maire de Rogiano Gravina et directeur du programme d'éducation pour adultes de Calabre, examine les plans d'une nouvelle école pour adultes. Rogiano Gravina, avec une population de 6000 habitants, est le plus actif de tous les villages de Calabre dans le domaine de la lutte contre l'illettrisme. Un millier d'enfants fréquentent les écoles publiques et $\mathbf{7 0 0}$ adultes viennent aux cours du soir financés par la Ligue pour la lutte contre l'analphabétisme, laquelle bénéficie du soutien du gouvernement. Il y a une pénurie de salles de classe et les étudiants adultes et paysans sont dispersés à travers tout le village dans $\mathbf{4 0}$ salles de classes - certaines d'entre elles se trouvent chez l'habitant - avec les instituteurs qui font la navette d'une salle à l'autre. L'enthousiasme des paysans est extraordinaire ; bon nombre d'entre eux doivent faire des kilomètres à pied depuis leur ferme éloignée pour arriver en classe, et puis rentrer chez eux à pied de nuit. Ces dernières années, le taux d'analphabétisme est passé de 68 à $22 \%$, et on a l'espoir que très bientôt Rogiano Gravina sera le premier village où l'analphabétisme aura été complètement éradiqué. Le Centre culturel de la Ligue est devenu le cœur de la vie du village. La méthode pédagogique est intéressante : 
bon nombre de soirées sont consacrées à la culture générale, avec des séances de questions-réponses. Un atelier rudimentaire permet aux jeunes gens du village de fabriquer des fournitures pédagogiques telles que des cartes de géographie, des meubles pour les salles de classe, du matériel de laboratoire de base, etc. Les instituteurs sont tenus en haute estime par la population et réservent certaines heures pour donner des conseils juridiques et aider les paysans dans leurs relations avec les autorités de l'État. Le médecin local donne des avis médicaux et des consultations au Centre culturel. Un orchestre, ainsi qu'un groupe de théâtre amateur, ont été montés au Centre.

11. LUTTE CONTRE L’ANALPHABÉTISME. Première leçon de lecture pour des paysannes de Rogiano Gravinia. L'analphabétisme est bien plus répandu chez les femmes que chez les hommes et la Ligue pour la lutte contre l'analphabétisme n'épargne pas ses efforts pour attirer les femmes aux cours du soir. En plus de la lecture et de l'écriture, on enseigne aux femmes la broderie et d'autres travaux manuels ; ainsi leurs cours du soir sont divisés entre la lecture, l'écriture et la couture. Broder les lettres de l'alphabet est une méthode à succès qui combine deux choses en une. Pour la lecture, le système de lettres mobiles qu'on aperçoit ici a prouvé son efficacité.

60 - Atelier de peinture au Centre de Rogiano Gravina. Les garçons préparent des cartes de l'Italie en plastique qui seront utilisées dans l'école de Rogiano Gravina, et plus tard, lorsque les besoins locaux auront été satisfaits, elles seront offertes aux écoles pauvres des environs.

64 - Vue d'ensemble de Rogiano Gravina.

68 - Une rue de Rogiano Gravina.

70 - Scène de rue à Rogiano Gravina. La petite fille au centre de la photo est en train de faire ses devoirs.

4. LUTTE CONTRE L'ANALPHABÉTISME. L'entrée du Centre de culture du peuple à Rogiano Gravina. Pendant la journée, les enfants vont à l'école ici ; le soir, on utilise les salles de classes pour l'éducation des adultes.

5. LUTTE CONTRE L'ANALPHABÉTISME. Une classe de $5^{\mathbf{e}}$ année primaire à Rogiano Gravina. C'est la dernière année d'enseignement obligatoire, qui se termine pour la plupart des enfants à l'âge de onze ans. À cet âge-là, beaucoup d'enfants travaillent déjà dans les champs pour aider leurs parents. Autrefois, leur courte période de scolarité était vite oubliée ; la campagne actuelle contre l'analphabétisme tente de résoudre ce problème en prolongeant l'éducation des jeunes avec des cours du soir, en créant des clubs pour la jeunesse, des ateliers, des bibliothèques, et en organisant des soirées dédiées à la culture générale pour que les jeunes continuent à s'instruire. 
6. LUTTE CONTRE L'ANALPHABÉTISME. Une classe de $5^{\mathbf{e}}$ année primaire à Rogiano Gravina. C'est la dernière année d'enseignement obligatoire, qui se termine pour la plupart des enfants à l'âge de onze ans. À cet âge-là, beaucoup d'enfants travaillent déjà dans les champs pour aider leurs parents. Autrefois, leur courte période de scolarité était vite oubliée ; la campagne actuelle contre l'analphabétisme tente de résoudre ce problème en prolongeant l'éducation des jeunes avec des cours du soir, en créant des clubs pour la jeunesse, des ateliers, des bibliothèques, et en organisant des soirées consacrées à la culture générale pour que les jeunes continuent à s’instruire.

83 - Entrée du Centre de Rogiano Gravina.

7. LUTTE CONTRE L'ANALPHABÉTISME. Une classe pour adultes au Centre culturel du peuple de Rogiano Gravina. L'instituteur guide la main hésitante d'un paysan âgé qui essaie d'écrire pour la première fois.

8. LUTTE CONTRE L'ANALPHABÉTISME. La première leçon d'écriture d'un paysan âgé au Centre culturel du peuple de Rogiano Gravina.

109 - Giuseppe Zanfini, directeur du Centre de Rogiano Gravina, aide une pauvre paysanne analphabète, Maria Viola, à remplir un document officiel. Lentement, les gens de Rogiano Gravina apprennent à faire confiance à leurs instituteurs. Ils savent à présent que le Centre est l'endroit où ils peuvent se rendre à chaque fois où ils ont besoin d'aide. Les instituteurs les accueillent avec joie, résolvent leurs problèmes, écrivent des lettres et remplissent des formulaires officiels, tout en expliquant patiemment l'importance de l'éducation. Une des raisons principales du succès rencontré à Rogiano Gravina repose sur l'idée que pour maintenir l'intérêt des gens pour l'éducation, il faut que l'école les aide. Les consultations juridiques, la rédaction de courriers, les soins médicaux, une petite bibliothèque, tout cela sert à maintenir l'intérêt des paysans pour l'école.

114 - Même légende que 109.

117 - Giuseppe Zanfini, directeur du Centre de Rogiano Gravina, est en train de lire une lettre qui vient d'arriver pour une paysanne illettrée, Emilia Tuoto. La lettre vient du fils d'Emilia, Nicola, qui s'est enfui de chez lui et a émigré en France trois ans plus tôt. Sa première lettre vient d'arriver après des années de silence, et Emilia est accourue au Centre pour se la faire lire. C'était une lettre très émouvante où son fils expliquait qu'il était parti dans l'espoir de trouver de meilleures conditions de travail, mais qu'il avait connu des moments difficiles ; maintenant que les choses allaient mieux, il demandait à sa mère de lui pardonner son silence, et de lui faire savoir comment il pourrait lui venir en aide. La première réaction d'Emilia a été de demander à 
être admise à l'école, et maintenant cette femme de 45 ans vient tous les soirs et travaille dur pour arriver à lire toute seule les lettres de son fils.

12. LUTTE CONTRE L'ANALPHABÉTISME. Le cours du soir de lecture pour les femmes. Le problème des étudiants qui ont de jeunes enfants est résolu de différentes manières. Parfois on les emmène à l'école et ils s'assoient aux côtés de leur mère ou de leur père. Dans le cas des nourrissons, la mère et le père s'en occupent à tour de rôle - un soir sur deux l'un reste à la maison tandis que l'autre va à l'école. Les anciennes traditions établies dans l'Italie du sud qui maintenaient la femme dans une situation très inférieure sont en train de changer ici.

13. LUTTE CONTRE L'ANALPHABÉTISME. Une jeune religieuse suit les cours du soir de la Ligue pour la lutte contre l’analphabétisme à Rogiano Gravina.

193 - Tout le monde reconnaît le besoin d'une réforme agraire en Italie du sud. Le Parlement italien examine en ce moment un projet de loi concernant la réforme agraire. Cependant, les paysans pauvres de Calabre ont pris les choses en main et se sont mis à occuper des terres en friche. Cette occupation n'est pas synonyme d'appropriation. Cela veut simplement dire que les paysans vont travailler la terre, faire la récolte, et donner au propriétaire sa part habituelle de la récolte, qui est de 30 à $40 \%$. Les paysans et travailleurs agricoles de Rogiano Gravina exploitent en moyenne un demi-hectare à un hectare (pour qu'une famille de paysans puisse s'assurer un niveau de vie acceptable, il faudrait qu'elle puisse exploiter au moins 6 à 8 hectares de terres). L'occupation des terres représente moins d'un demi-hectare par famille et ne résout pas les problèmes de subsistance de base. L'occupation est pacifique, sans aucune présence de policiers ou carabiniers. L'homme qui est au centre de la photo, Oreste Rossini, a une famille de cinq personnes, une exploitation foncière d'un peu plus d'un hectare, et est un élève très actif aux cours du soir du Centre.

145-149 - Images de l'occupation des terres.

158 - L'école primaire de Cimino, près du village de San Marco dans la province de Cosenza. Une petite étable transformée en école avec une porte en guise de fenêtre. Vingt-cinq enfants environ fréquentent l'école.

161 - $\quad$ Arrivée des enfants à l'école à Cimino.

168 - Une des nombreuses photos de femmes portant divers fardeaux sur la tête. J'ai l'idée que l'on pourrait utiliser ces photos comme introduction symbolique à l'histoire. Je n'ai pas encore réussi à trouver la formule exacte, mais l'idée générale c'est que les gens de Calabre se sont 
jusqu'ici servis de leur tête pour porter des charges, mais découvrent maintenant qu'ils peuvent se servir de leur tête pour leur éducation.

21. LUTTE CONTRE L'ANALPHABÉTISME. Intérieur du bâtiment scolaire de Cimino, près du village de San Marco dans la province de Casenzo. Cette petite étable sans fenêtre a été transformée en école ; la seule lumière vient par la porte ouverte. Vingt-cinq enfants fréquentent l'école, et l'institutrice, Olga Natale, se plaint des conditions - le toit fuit et le vent froid souffle dans toute la pièce. Par la porte ouverte on peut apercevoir un jeune berger qui doit s'occuper de ses moutons et ne peut pas fréquenter l'école.

Intérieur de l'école à Cimino. L’institutrice est assise tout près de la porte. Elle s'appelle Olga Natale, et elle se plaint beaucoup des conditions de l'école avec un toit qui fuit et le vent et le froid qui soufflent dans toute la pièce. Par la porte ouverte de l'école on aperçoit un jeune berger et ses moutons. Pour moi, cette photo exprime un des problèmes de l'éducation en Calabre : les enfants doivent travailler très jeunes et n'ont pas les moyens d'aller à l'école.

178-180 - Jeune berger et ses moutons près de Cimino.

22. LUTTE CONTRE L'ANALPHABÉTISME. Ce petit berger de Cimino a commencé à travailler alors qu'il était encore d'âge scolaire et il ne peut pas aller à l'école.

193 - Cours du soir à l'école populaire de San Nicola da Crissa, un village pauvre de montagne dans la province de Catanzaro.

197 - École primaire de San Nicola da Crissa.

210 - Un père et son enfant à San Nicola da Crissa.

219 - Une vieille carte de l'Europe toute déchirée dans une salle de classe à Bagaladi.

224 - Le chemin muletier menant au village de montagne de Saucci, dans la province de Reggio Calabria. De nombreux villages en Italie du sud n'ont pas de routes et ne sont pas faciles d'accès. On arrive à Saucci, un petit village de bergers, par un chemin muletier long de trois kilomètres qui donne sur la route principale, bloquée par la neige pendant presque deux mois par an.

226 - L'école de Saucci est importante pour la région, et, beaucoup d'enfants sont obligés de faire trois ou quatre kilomètres à pied par des chemins de montagne pour pouvoir aller à l'école. L'instituteur, Antonio Janni, né aux États-Unis de parents italiens, est revenu en Italie il y a quinze ans, et, une fois enrôlé dans l'armée italienne, a perdu sa nationalité américaine. C'est sa première année à l'école de Saucci. Il a hérité du travail de son cousin qui avait attrapé une pneumonie et qui est décédé probablement d'épuisement. 
Ce n'est pas étonnant car ce travail est dur. Il n’y a pas de logement pour lui à Saucci. Il vit à Bagaladi, à $16 \mathrm{~km}$ de là. Tous les matins il emprunte avec son scooter la route de montagne à lacets, tortueuse et extrêmement dangereuse par mauvais temps, jusqu'au croisement où débute le sentier muletier qui mène à Saucci. Bien souvent il transporte un lourd colis de ravitaillement pour les enfants de l'école, parce qu'il n'y a personne d'autre pour livrer la nourriture fournie par le gouvernement et l'UNICEF. Janni enseigne, sert les repas, conseille les villageois de Saucci et représente le principal contact du village avec le monde extérieur. Et plus tard dans l'année, quand les journées se rallongent et qu'il ne doit pas se dépêcher de rentrer chez lui avant que l'obscurité ne rende la route impraticable, il s'attarde pour enseigner aux adultes la lecture et l'écriture.

24. LUTTE CONTRE L'ANALPHABÉTISME. Un exemple de bâtiment scolaire rudimentaire en Calabre. Celui-ci, situé à Capistrano, est au-dessus d'une étable.

18. LUTTE CONTRE L'ANALPHABÉTISME. Antonio Janni bavarde avec ses élèves pendant la pause déjeuner. Après l'école, il a coutume de rester à Saucci pour apprendre aux adultes à lire et à écrire.

191 - L'école à Cimino.

190 - Jeune garçon revenant du travail.

236 - Salle de classe à Saucci. Notez les sandales faites avec de vieux pneus.

19. LUTTE CONTRE L'ANALPHABÉTISME. Les enfants de Saucci vont à l'école en suivant les sentiers de montagne. Ils font souvent des kilomètres à pied depuis les villages voisins pour pouvoir aller à l'école. En hiver, les sentiers sont très dangereux.

20. LUTTE CONTRE L'ANALPHABÉTISME. Les écoles sont rares en Calabre, les enfants sont souvent obligés de faire des kilomètres à pied pour rejoindre les villages où se trouvent les écoles.

262 - Écoliers en route pour l'école sur des sentiers de montagne.

256 - Intérieur d'une maison de paysans à Saucci.

259 - Four à pain rudimentaire devant une maison à Saucci. Ils cuisent le pain une fois par semaine.

299 - En chemin pour Capistrano, dans la province de Catanzaro. Misérables habitations de paysans. Le four à pain est au milieu.

276 - Une vue de Saucci avec les nuages recouvrant les sommets des montagnes. 
278 - Des écoliers poussent le scooter de leur instituteur, Antonio Janni, avant qu'il ne démarre sur la route de Bagaladi.

281 - Écoliers sur le long chemin du retour à la maison.

14. LUTTE CONTRE L'ANALPHABÉTISME. La vie des instituteurs en Calabre et très dure. Antonio Janni, né aux États-Unis de parents italiens, est revenu en Italie il y a quinze ans, et, une fois enrôlé dans l'armée italienne, a perdu sa nationalité américaine. C'est sa première année à l'école de Saucci. Il a hérité du travail de son cousin qui avait attrapé une pneumonie et qui est décédé probablement d'épuisement. Ce n'est pas étonnant, car ce travail est dur. Il n'y a pas de logement pour lui à Saucci. Il vit à Bagaladi, à $16 \mathrm{~km}$ de là. Tous les matins il emprunte avec son scooter la route de montagne à lacets, tortueuse et extrêmement dangereuse par mauvais temps, jusqu'au croisement où débute le sentier muletier qui mène à Saucci. Bien souvent il transporte un lourd colis de ravitaillement pour les enfants de l'école, parce qu'il n'y a personne d'autre pour livrer la nourriture fournie par le gouvernement et l'UNICEF. Janni enseigne, sert les repas, conseille les villageois de Saucci et représente le principal contact du village avec le monde extérieur. Et plus tard dans l'année, quand les journées se rallongent et qu'il ne doit pas se dépêcher de rentrer chez lui avant que l'obscurité ne rende la route impraticable, il s'attarde pour enseigner aux adultes la lecture et l'écriture.

309 - Misérables habitations de paysans sur la route de San Nicola da Crissa.

25. LUTTE CONTRE L'ANALPHABÉTISME. Un des problèmes majeurs que rencontre la campagne contre l'illettrisme est celui du travail des enfants en Calabre.

315 - $\quad$ En Calabre, les femmes se servent de leur tête pour porter des charges.

324 - Les enfants aussi.

319 - Une famille au travail dans son champ.

328-330 - Photos de transport de fardeaux.

334 - $\quad$ Enfants bergers sur la route de Spezzano Albanese.

345 - $\quad$ Elève berger au cours du soir de Rogiano Gravina.

346 - $\quad$ Oreste Rossini (voir photo 139) assiste au cours du soir.

338 - Cours du soir à Rogiano Gravina. Oreste Rossini sur la gauche.

9. LUTTE CONTRE L'ANALPHABÉTISME. Leçon de lecture d'un paysan. Un jeune berger (à l'arrière-plan) fréquente les cours avec les adultes. Quand il était petit, il ne pouvait pas aller à l'école parce qu'il devait garder son troupeau ; maintenant il peut lui aussi bénéficier des nouveaux cours du soir et il apprend à lire et à écrire. 
10. LUTTE CONTRE L'ANALPHABÉTISME. Gros plan d'un jeune berger dans un cours pour adultes. (Voir photo 9). Quand il était petit, il ne pouvait pas aller à l'école parce qu'il devait garder son troupeau ; maintenant il peut lui aussi bénéficier des nouveaux cours du soir et il apprend à lire et à écrire.

349-352 - Paysage calabrais. Le village de Morano Calabro.

15. LUTTE CONTRE L'ANALPHABÉTISME. Le chemin muletier menant au village de montagne de Saucci dans la province de Reggio Calabria. De nombreux villages en Italie du sud n'ont pas de routes et ne sont pas faciles d'accès. On arrive à Saucci, un petit village de bergers, par ce chemin muletier long de trois kilomètres qui est rattaché à la route principale, laquelle est bloquée par la neige pendant presque deux mois par an.

16. LUTTE CONTRE L'ANALPHABÉTISME. Vue du village de Saucci, avec les nuages qui recouvrent les sommets des montagnes. Saucci, comme bien des villages de montagne en Italie du sud, n'est pas facile d'accès. On y accède par un chemin muletier de trois kilomètres de long qui est rattaché à la route principale, laquelle est bloquée par la neige pendant presque deux mois par an.

LES PHOTOS AU LEICA SONT SURTOUT DES DOUBLES DES PHOTOS PRISES AU ROLLEI. VOUS TROUVEREZ PLUS DE DESCRIPTIONS DÉTAILLEES AVEC LES PLANCHES-CONTACT DU ROLLEI. VOICI QUELQUES REMARQUES SUPPLÉMENTAIRES.

\section{LEICA 1}

7 - $\quad$ Misérables habitations de paysans sur la route de Capistrano.

22 - École primaire à San Nicola da Crissa.

\section{LEICA 7}

\section{OCCUPATION DES TERRES À ROGIANO GRAVINA}

17 - Femme enceinte au travail pendant l'occupation des terres.

10 - Femme âgée au travail.

22 - Les paysans mangent un pain grossier pour leur repas de midi. Ils m'ont montré leur pain : il était dense, humide et fait d'un mélange de farine de maïs et d'un petit peu de farine de blé.

\section{$\underline{\text { LEICA } 3}$}

3 - L'école de Capistrano est située au-dessus d'une étable et pendant les journées chaudes et ensoleillées, l'odeur est épouvantable.

25 - Enfants allant à l'école sur les chemins de montagnes à Saucci.

15 - Vue de Saucci. 


\section{LEICA 5}

24-34 - Occupation des terres à Rogiano Gravina.

17 - Écolier marchant le long des rails de chemin de fer. J'ai essayé de dramatiser cette longue marche vers l'école sur une route qui traverse la montagne et longe les rails.

6 - Intérieur de l'école de Cimino. 



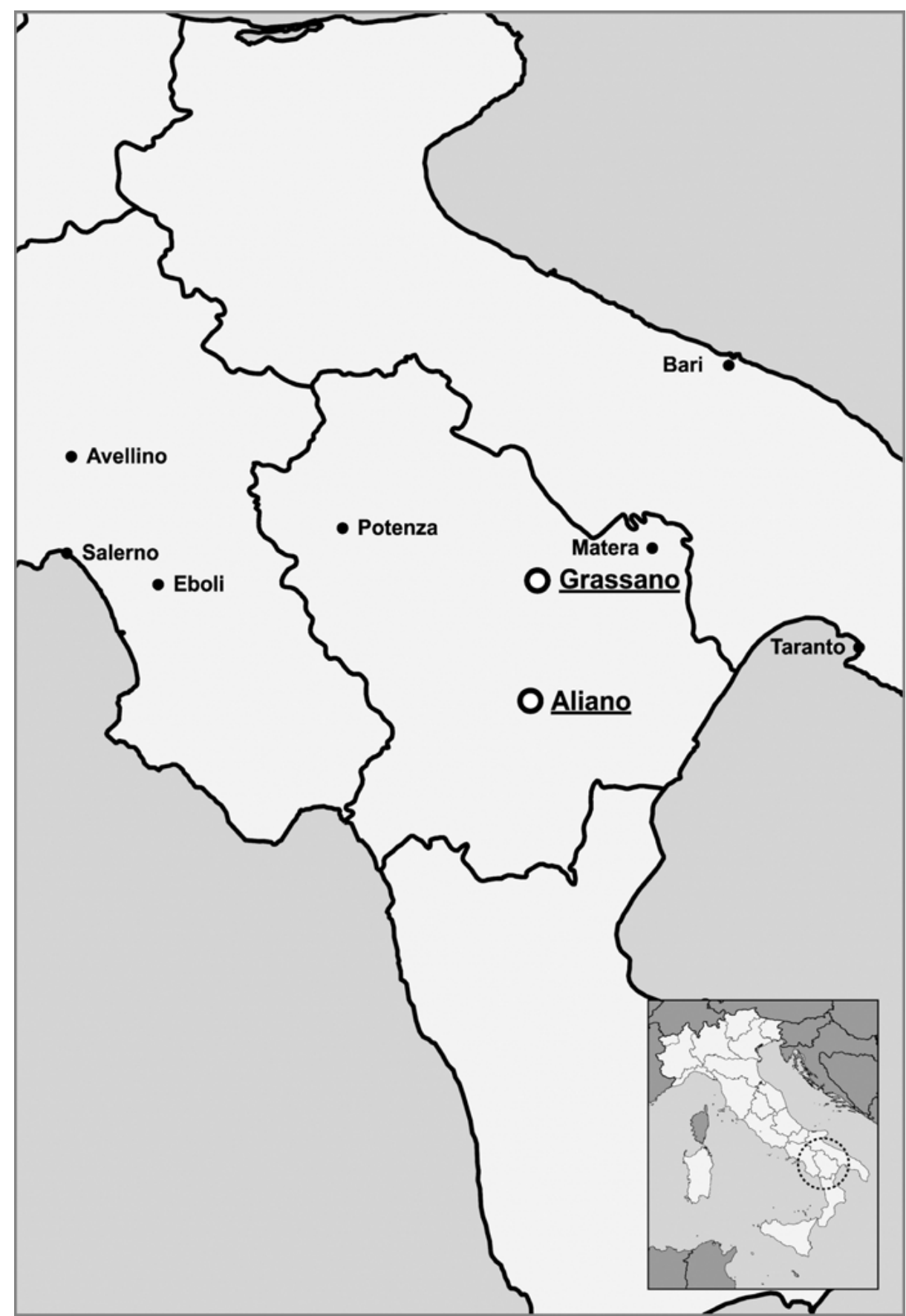

Fig. 2.6 Carlo Levi's places of exile (confino) in 1935-1936 / Lieux de l'exil (confino) de Carlo Levi en 1935-1936 @ Thomas Amen. 


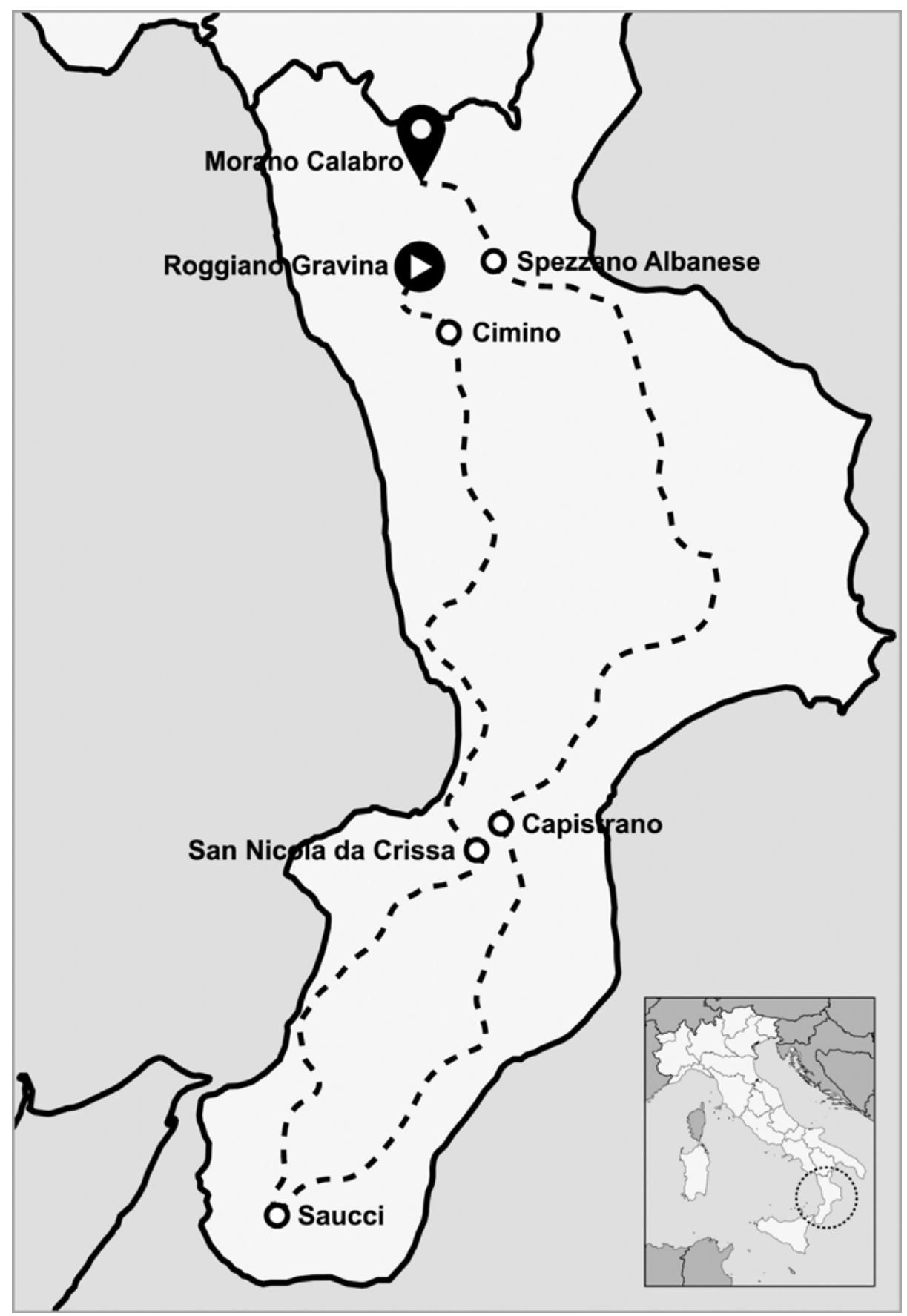

Fig. 2.7 David “Chim” Seymour’s itinerary in 1950 / Itinéraire de David « Chim » Seymour en 1950 ㄷ) Thomas Amen. 
